\title{
The Quantum Hall Effect of Field Induced Spin Density Wave Phases: the Physics of the Ultra Quantum Crystal
}

\author{
Pascal Lederer \\ Physique des Solides,Université Paris-Sud, F91405 Orsay-cedex, France \\ Laboratoire associé au CNRS
}

May 27, 1996

\begin{abstract}
The Quantum Hall Effect of Field Induced Spin Density Waves is accounted for within a weak coupling theory which assumes that in the relevant low temperature part of the phase diagram the quasi one dimensional conductor is well decribed by Fermi liquid theory. Recent experimental results show that sign inversion of the Hall plateaux takes place all the way down from the instability line of the normal state. The Quantum Nesting Model, when it takes into account small perturbations away from perfect nesting, describes well, not only the usual sequence of Hall Plateaux, but also the anomalies connected with sign inversion of the Hall Effect. Experimental observation of de-doubling of sub-phase to subphase transition lines suggests that superposition of SDW order parameters occurs in some parts of the phase diagram. The collective elementary excitations of the Ultra Quantum Crystal have a specific magneto-roton structure. The SDW case exhibits, apart from the usual spin waves, topological excitations which are either skyrmions or half skyrmions. It is suggested that magneto-rotons may have been observed some years ago in specific heat experiments.
\end{abstract}

Pacs numbers 72.15.Nj 73.40.Hm 75.30.Fv. 75.40.Gb

\section{Introduction}

A step-like Hall voltage behaviour under field was found in the strongly anisotropic quasi one dimensional compound $\left(\mathrm{TMTSF}_{2} \mathrm{ClO}_{4}\right.$ [迆] very shortly after the first experimental hints of a cascade of phase transitions in quasi-1D conductors under magnetic field were published [2]. It was discussed in the experimental paper in terms of the Quantum Hall Effect[3].

In this review paper, I shall discuss the present theoretical understanding of this phenomenon, and of some related aspects of the physics of Field Induced Spin Density Wave phases. The material includes known results dating back to 1984, more recent work, some of which still unpublished at the time I am writing, and new results, not published elsewhere. A review dealing with the theory of Fiel Induced Density Waves up to 1991 can be found in ref. [禺.

Within a weak coupling approach, Gor'kov and Lebed pointed out the crucial appearance, under an applied magnetic field $\mathrm{H}$, of a logarithmic divergence in the (one loop) 
spin staggered static susceptibility $\chi_{0}\left(2 k_{F}, T, H\right)$ because of the open quasi nested Fermi surface [5]. In quasi classical terms, the electron orbits become one dimensional under magnetic field and this restores the 1-D logarithmic divergence of the (bare) spin susceptibility. Gor'kov and Lebed discarded an interpretation of the Hall plateaux in terms of the Quantum Hall Effect [3], because, at the time, there was no sign of any significant decrease of the longitudinal resistivity coinciding with the Hall plateaux.

They pointed out the thermodynamic nature of the phenomenon, which they described as a cascade of phase transitions with periodic re-entrance of the normal phase between two identical Spin Density Wave phases.

However, shortly after the work by Gor'kov and Lebed [5], Héritier, Montambaux and Lederer [6] suggested that in fact the step-like Hall voltage was indeed a new form of Quantized Hall Effect, intimately connected with the cascade mechanism. Their argument was based on the discovery that the most divergent loop, in the presence of the magnetic field, is obtained for a quantized, field dependent longitudinal wave vector

$$
\mathbf{q}=\left(k_{x}=2 k_{F}+n\left(2 \pi / x_{0}\right), k_{y} \simeq \pi / b, k_{z}=\pi / c\right) .
$$

In this expression, the length $x_{0}$ is the magnetic length. This length appears naturally if one considers the area $b x_{0}$ threaded by one flux quantum $\phi_{0}$ between two neighbouring chains at a distance $b$ under a field $\mathrm{H}$ :

$b x_{0} H=\phi_{0}=h /|e|$

$\left(x_{0}=h /(e b H)\right.$ is of order $100 \mathrm{~nm}$ if $H \simeq 10$ Teslas). In the following, I shall use the notation $G=2 \pi / x_{0}$ for the wave vector associated with $x_{0}$. There is also an energy scale associated with this length, $\hbar \omega_{c}=\hbar v_{F} G / 2=e v_{F} b H / 2$. The logarithmic growth of the staggered susceptibility occurs when $k_{B} T<<\omega_{c}$.

According to Héritier, Montambaux and Lederer [6], the index $\mathrm{n}$ appearing in the wave vector $\mathrm{x}$ component of the Spin Density Wave (SDW) instability labels each SDW subphase and decreases by one unit from subphase to subphase as $\mathrm{H}$ increases. At the same time, this index $\mathrm{n}$ is the number of exactly filled Landau levels (Landau bands in fact, as will be discussed later on) of unpaired quasiparticles left by the SDW condensation in a situation of imperfect nesting. When $\mathrm{H}$ changes, a competition develops between the condensation energy and the diamagnetic energy: the former is lowered if electrons and holes condense and increase the order parameter; the latter is lowered if Landau levels are exactly filled and the Fermi level sits between two Landau levels; accordingly, the SDW wave vector changes, at fixed n, so that the pockets of unpaired particles have exactly the right area for an integer number of filled Landau levels below the Fermi level. The SDW wavevector varies smoothly with, say, increasing field until it becomes energetically favourable to jump to the next quantum number (n-1). This picture was later on confirmed by the analytic theory of the condensed phase, with an order parameter described by a single Fourier component of the staggered magnetization [7]. The general structure of the phase diagram was also studied independently by Lebed [8], with similar result. A way of formulating this picture is to describe the quantization condition as a nesting quantization: the area between one sheet of the normal state Fermi surface and the other sheet translated by $\mathbf{q}$ is quantized in terms of the area quantum $e H / \hbar$, leading to the condition 1. Hence the name "Quantized Nesting Model" (QNM) dubbed by the authors of ref. [6]. The Quantized Hall Effect of FISDW phases is thus a special example of a general result due to Halperin, following whom the integer quantum Hall effect should be 
observed in a bulk system in a magnetic field if the chemical potential lies in an energy gap [9].

There is now overwhelming evidence for the thermodynamic nature of the cascade of Field Induced SDW (FISDW) phases [10], and for the occurrence of a novel type of QHE in those phases [11]. Both aspects are intimately connected. Very well defined Hall plateaux with the ratios 1:2:3:4:5 are observed in $(T M T S F)_{2} P F_{6}$, for example, where the ratio of the resistivity tensor components $\rho_{x y} / \rho_{x x}$ can be as large as 75 within a plateau, and strongly decreases in the narrow region between the plateaux 11]. Figure 1 is a typical QHE curve obtained in $(T M T S F)_{2} P F_{6}$.

In this paper, I discuss the problems that have arisen in the theoretical picture because of progress in experiments over the last few years; two main phenomena have led to conflicting views: the "Ribault anomaly" and the fine structure of the phase diagram. The former is the observation that under certain conditions, for example for certain values of the applied pressure, and (in the case of the $\mathrm{ClO}_{4}$ compound), for a very slow cooling rate, the FISDW exhibits a change of sign of the Hall plateau 12] [13]. The latter is the interpretation of specific heat anomalies within the domain of existence of the FISDW in terms of "arborescence" of the phase diagram 14] [15] [16].

It turns out that recent experiments have helped in clarifying our understanding both of the "Ribault anomaly" [13] and of the nature of the phase diagram 150 16. In particular, following the important experiments by Balicas, Kriza and Williams [13], Zanchi and Montambaux have shown that the sign reversal of the QHE can be described within the Quantized Nesting Model (the "Standard Model") at the cost of minor conceptual changes [17. This contrasts with repeated statements in the literature denying the possibility of accounting for this phenomenon within the "standard model" [18], or with repulsive electron-electron interactions alone[19].

FISDW are both a particular example of electron-hole condensate describable as a quantum crystal, and a novel manifestation of quantum orbital resonances. As such, their collective excitations are expected to exhibit specific features. Besides the usual Goldstone bosons, phasons and spin waves, which appear as a result of the various broken continuous symmetries in the FISDW (translation symmetry and spin rotational invariance) I shall discuss the occurrence of the magneto-roton[3], the existence of which in FISDW was discussed and proved by Lederer and Poilblanc, [20], and the skyrmion (and half-skyrmion), discussed in this context by Yakovenko [19]

The point of view adopted in this paper is that the Quantum Hall Effect observed in the FISDW phases is reasonably well described within a weak coupling approach : I assume that the normal state in the absence of magnetic field is an anisotropic Fermi liquid with quasi 1D Fermi sheets; the electronic hopping in all three directions at sufficiently low temperature is coherent. Since all the interesting physics occurs at temperatures smaller than $t_{c} / k_{B}$ (where $t_{c}$ is the smallest interchain hopping, along the field direction) this is a reasonable assumption. Furthermore, since I consider situations where the magnetic field is orthogonal to the most conducting plane, hopping along that direction will remain coherent in all cases. Other interesting and complicated situations may arise in a different field geometry, if electronic interactions are sufficiently strong. 21] 


\section{The Quantum Hall Effect of Field Induced SDW Phases}

I first recall the results obtained when the order parameter is described by a single Fourier component of the magnetization. For simplicity, I will restrict the discussion to the case of a transverse magnetization, such that the Zeeman term plays no role: the magnetic field is in the $\mathrm{z}$ direction, perpendicular to the most conducting plane, the magnetization which appears as a result of the orbital effect and of electron-hole pairing is lying in the $(\mathrm{x}, \mathrm{y})$ plane.

\subsection{The Quantized Nesting Model}

Consider a simple model of an orthorombic, anisotropic quasi two-dimensional conductor. The open Fermi surface is described by the following dispersion relation, linearized around the Fermi level in the longitudinal direction:

$$
\begin{aligned}
\epsilon(\mathbf{k}) & =v_{F}\left(\left|k_{x}\right|-k_{F}\right)+\epsilon_{\perp}(\mathbf{k}) \\
\epsilon_{\perp}(\mathbf{k}) & =-2 t_{b} \cos k_{y} b-2 t_{b}^{\prime} \cos 2 k_{y} b-2 t_{c} \cos k_{z} c
\end{aligned}
$$

In the transverse $b$ direction, a second harmonic is introduced to take into account the violation of perfect nesting, defined by the condition $\epsilon(\mathbf{k})=-\epsilon(\mathbf{k}+\mathbf{Q})$. If $t_{b}^{\prime}$ vanishes, this equation holds with $\mathbf{Q}=\left(k_{F}, \pi / b, \pi / c\right)$. The existence of a non zero $t_{b}^{\prime}$ arises from linearization of the dispersion relation along the $x$ direction [22] and/or from next nearest neighbour coupling between chains [23]. Perfect nesting in the $\mathrm{z}$ direction makes the problem effectively two dimensional. The magnetic field, parallel to the $c$ direction is described by the vector potential $\mathbf{A}=(0, H x, 0)$. This choice of gauge is crucial to simplify the problem and let the effective one dimensionnality nature of the problem appear in the simplest fashion. The electron transfer integrals along the three crystal axes have the following orders of magnitude:

$$
\begin{gathered}
t_{a} \simeq v_{F} k_{F} \simeq 300 \mathrm{meV}>>t_{b} \simeq 30 \mathrm{meV} \\
t_{b}>>t_{b}^{\prime} \simeq t_{c} \simeq 1 \mathrm{meV}
\end{gathered}
$$

The equations (à la Gor'kov) which describe the ordered phase are as follows:

$$
\begin{aligned}
\left(i \omega_{n}+i v_{F} \frac{d}{d x}\right) g+\tilde{\Delta} f & =\delta\left(x-x^{\prime}\right), \\
\left(i \omega_{n}-i v_{F} \frac{d}{d x}-N v_{F} / x_{0}\right) f+\tilde{\Delta}^{\star} g & =0,
\end{aligned}
$$

where $g$ and $f$ are diagonal and off-diagonal parts of the Green function, the phases of which have been properly defined [24] [7].

$$
\begin{aligned}
\tilde{\Delta}(x) & =\Delta \Sigma_{n} I_{n} \exp \left(-i n x / x_{0}+i \Phi_{n}\right), \\
\Phi_{n} & =n p-z \sin p-z^{\prime} \sin 2 p \\
I_{n} & =\Sigma_{p} J_{n-2 p}(z) J_{p}\left(z^{\prime}\right) \\
z & =\left(4 t_{b} x_{0} / v_{F}\right) \cos Q_{t} b / 2 \\
z^{\prime} & =\left(2 t_{b}^{\prime} x_{0} / v_{F}\right) \cos Q_{t} b
\end{aligned}
$$


and $J_{p}(z)$ is the pth-order Bessel function of argument z. $\Delta$, the order parameter, and the wave vector $Q=\left(Q_{\|}, Q_{t}, \pi / c\right)$ are determined self-consistently so as to minimize the free energy. We know that $Q_{\|}=2 k_{F}+N G$. $\tilde{\Delta}(x)$ acts as an effective potential which couples electronic states not only at $\mathbf{k}$ and $\mathbf{k}+\mathbf{Q}$ because of SDW ordering but also $\mathbf{k}$ and $\mathbf{k}+\mathbf{Q}-N G\left(\hat{\mathbf{i}} k_{x} /\left|k_{x}\right|\right)$. Therefore, the quasi particle spectrum exhibits a series of gaps [7] $\Delta_{n}=\Delta I_{N}$ opened at $k= \pm(1 / 2)\left(Q_{\|}-N G\right)$. The free energy is minimum when the Fermi level lies in the middle of the largest of these gaps $\Delta_{N}=\Delta I_{N}$. This occurs when $Q_{\|}(H)=2 k_{F}+N G$. The gaps result from density wave ordering and orbital quantization: at the level of the one particle Green function, the orbital periodicity acts as a broken translational symmetry, which vanishes at the level of two particle Green function, and density density correlation function; this feature, which is a specific expression of gauge invariance in this problem gives rise to the magneto-roton minimum, as will be discussed later. We thus have separate Landau bands containing $1 / 2 \pi b x_{0}=e H / h$ states per unit surface. The distance in energy between the centers of two neighbouring Landau bands is $\hbar \omega_{c}$. At zero temperature, each quantized SDW phase has either completely filled or completely empty Landau bands. No FISDW phase can exist at temperatures $T \geq \hbar \omega_{c}$

If the Spin Density Wave is pinned by some mechanism or other (say impurities), only single particle excitations contribute to the conductivity. Since perfect nesting along the $\mathrm{z}$ direction makes the problem effectively two dimensional, Laughlin's gauge invariance arguments [3] tell us that the single atomic layer Hall conductivity is exactly quantized at zero temperature in units of $e^{2} / h$, i.e. we must have

$$
\sigma_{x y}=n e^{2} / h
$$

The value of $n$ is precisely the value $N$ which labels the FISDW subphase where $Q_{\|}-2 k_{F}=$ $N G$. The proof was given by Poilblanc et al. 25] using an approach due to Streda 26]. Following the latter, at fixed chemical potential $\mu$ and in a field independent potential,

$$
\begin{aligned}
\sigma_{x y} & =e \partial \rho(\mu, B, \Delta, \mathbf{Q}) /\left.\partial B\right|_{\Delta, \mathbf{Q}} \\
\rho(\mu, B, \Delta, \mathbf{Q}) & =\int_{-\infty}^{\mu} d \eta \operatorname{Tr} \delta(\eta-H(B, \Delta, \mathbf{Q}))
\end{aligned}
$$

where $H$ is the Hamiltonian. Taking into account the $B$ dependence of $Q_{\|}$, and noticing that $\rho$ does not depend on $\Delta$ and $Q_{\perp}$ when $\mathbf{Q}=\mathbf{Q}_{\mathbf{N}}$, i.e. when the Fermi level lies in a gap , St $\breve{r}$ eda's formula in the present case reads:

$$
\sigma_{x y}=-\left.e \frac{\partial Q_{\|}}{\partial B} \frac{\partial \rho}{\partial Q_{x}}\right|_{\Delta=\Delta(B, T), \mathbf{Q}=\mathbf{Q}_{\mathbf{N}}}
$$

Since $Q_{\|}^{N}(B, \rho)=2 k_{F}(\rho)+N G=2 k_{F}(\rho)+N|e| B b / \hbar$, we get $\partial Q_{\|}^{N} / \partial B=N|e| b / \hbar$. since $\partial \rho / \partial Q_{x}=1 / 2 \pi b$, we have eventually:

$$
\sigma_{x y}=N e^{2} / h
$$

This result was rederived by Yakovenko[19] using Kubo formula (which is also at the basis of Strreda's work) [27]. He used the topological properties of the wave functions in reciprocal space which result from the variation of their phase factor upon transporting them along closed contours. He argued that in fact the quantum number $N$, which results from his analysis if spinless electron-hole pairing (i.e. CDW pairing) is taken into account 
should be replaced by $2 N$ in the case of SDW pairing. The ratio of QHE plateaux conductivities is the accurately determined quantity in experiments, since the number of layers of a given sample is ill determined.

Thus, the theory based on the notion that the FISDW order parameter is well described by a single harmonic accounts in a satisfactory way for Hall experiments conducted in the Bechgaard salt $P F_{6}$ compound [11]. This is strong evidence in favour of the new Quantum Hall Effect mechanism described in [6].

\subsection{The sign reversals of the Quantum Hall Effect}

One physical effect, however, is conspicuously out of the picture described in the last section: the reversal of the sign of the Hall effect, which was first discovered by Ribault: under certain conditions of thermal preparation, a few "negative" (by convention) plateaux may appear in $(T M T S F)_{2} \mathrm{ClO}_{4}$ when the field varies. Such sign reversals have been found to occur under certain circumstances (pressure, cooling rates, and so on), in limited field range in the $\mathrm{ClO}_{4}, \mathrm{PF}_{6}$ [28] [29] and in the $\mathrm{ReO}_{4}$ compound [30]

A significant progress was achieved recently when Balicas Kriza and Williams [13] reported a negative Hall plateau ( with quantum number -2), inserted between two positive ones (with quantum number 3 and 2 ) which they could follow all the way from low temperatures to the critical line separating the FISDW phase from the normal state. Figure 2 depicts the experimental results. The crucial observation is that the negative Hall plateau may arise continuously, via a second order phase transition, from the normal state, in a finite interval of magnetic field. Indeed, this observation means that the FISDW phase with negative quantum number arises from divergent fluctuations of the normal phase, at a wave vector $Q_{x}=2 k_{F}-G$. It means that no mechanism based on free energy expansions to high order in the order parameter is able to account for this phenomenon: the harmonic term alone already contains the sign change, which must be a property of the bare spin susceptibility! This observation was a puzzle, because analytic and numerical work on the bare static spin susceptibility $\chi_{0}(\mathbf{q}, H)$ within the Quantized Nesting Model described in the previous section showed that the logarithmic divergences at wave vectors with "negative" quantum numbers have smaller amplitude than the "positive" ones. In other words, within the standard model, the normal phase instability to positive Hall plateaux always overcomes the transition to negative ones [31] 32].

A very simple clue to this puzzle was given very recently by Zanchi and Montambaux 17]. They pointed out that the competition between logarithmic divergences in $\chi_{0}(\mathbf{q}, B)$ at positive and negative quantum numbers depends on small perturbation terms which arise from higher order harmonics of the expansion in Fourier series of the dispersion relation in equ. 2. They investigated the following dispersion relation (setting $k_{y} b=p$ )

$$
-\epsilon_{\perp}=2 t_{b} \cos p+2 t_{b}^{\prime} \cos 2 p+2 t_{3} \cos 3 p+2 t_{4} \cos 4 p
$$

The calculation of the normal metal-FISDW instability line boils down to the determination of the absolute maximum of

$$
\chi_{0}(\mathbf{Q}, H)=\Sigma_{n} I_{n}^{2}\left(Q_{\perp}\right) \chi_{0}^{1 D}\left[Q_{\|}-n / x_{0}\right]
$$

This expression exhibits the structure of $\chi_{0}$ as the sum of one dimensional terms $\chi_{0}^{1 D}$ shifted by the magnetic field wave vector $G=e H b / \hbar$ [33]. Figure 3 exhibits the behaviour 
of $\chi_{0}$ at $q_{z}=\pi / c$ when $t_{3}=t_{4}=0$. In equ. 11, the coefficient $I_{n}$ depends on the dispersion relation:

$$
I_{n}\left(Q_{\perp}\right)=<\exp i\left[T_{\perp}\left(p+Q_{\perp} / 2\right)+T_{\perp}\left(p-Q_{\perp} / 2+n p\right)\right]>
$$

where $T_{\perp}(p)=\left(1 / \hbar \omega_{c}\right) \int_{0}^{p} t_{\perp}\left(p^{\prime}\right) d p^{\prime}$ and $<\ldots>$ denotes the average over $\mathrm{p}$.

The third harmonic term in equ.10, $t_{3}$ is found, if sufficient (in practice $t_{3} \geq 0.2 t_{b}^{\prime}$ ), to perturb nesting so that two degenerate maxima at even quantum numbers can become the absolute maxima. Those correspond to $Q_{\perp}=\pi / b$ and $Q_{\|}-2 k_{F}= \pm N G$. At odd quantum number, the absolute maximum is non degenerate and corresponds to positive $N$. The fourth harmonic term lifts the degeneracy between the two degenerate maxima with even quantum numbers; on the $Q_{\perp} 1$ ine,

$$
I_{ \pm N}(\pi / b)=<\exp \left[\frac{4 i}{\hbar \omega_{c}}\left(t_{b}^{\prime} \sin 2 p \pm \frac{t_{4}}{2} \sin 4 p\right)+i N p\right]>
$$

If $N$ is odd, $I_{N}=0$; If $N$ is even, $I_{-|N|}^{2}>I_{|N|}^{2}$ so that a phase with negative even $N$ is favoured, and a sequence of positive and negative Hall numbers is obtained, in a way which reproduces experimental results quite satisfactorily, with quite reasonable values of $t_{4} \simeq .025 K$. This in turn explains why the landscape of Hall plateaux depends so sensitively on pressure. Figure 4 shows how $\chi_{0}$ at $q_{z}=0$ changes when non zero $t_{3}$ and $t_{4}$ are taken into account.

In view of this, it appears that the standard model, suitably completed with small perturbing terms, provides an adequate basis to understand the long standing puzzle of Quantum Hall sign reversals, ( the Ribault anomaly), without resorting to a qualitatively different picture.

\subsection{Multiple Order Parameters}

It was noted from the start [7] that the self consistency condition for the FISDW order parameter opened the way for multiple order parameters solutions, with a superposition of Fourier components of the magnetization. This can happen 1) at low temperatures, when anharmonic terms in the expansion of the free energy come into play, since those might possibly make the coupling between order parameters attractive and 2) close to the phase boundaries between two subphases of the standard model, where two fluctuation modes of the non interacting electron gas diverge simultaneously, at quantum numbers $N$ and $N+1$ [34]. 35].

Theoretical investigations [36] [18] of such complex solutions have been triggered by experimental reports of a complex, branched, tree-like or arborescent phase diagram at low temperatures [14]. This complexity has been thought to be associated to the sign change in the Hall effect [18]. The latter was ascribed to discreteness effects of the lattice along the chains, which is not taken into account in the standard model. A periodic lattice potential is known, in a non interacting electron gas, to lead to an irregular pattern of positive and negative integer Hall plateaux, due to the complexity of the electronic structure of the two dimensional isotropic periodic lattice under magnetic field 38]. The notion that the latter could be relevant to the situation of highly anisotropic conductors such as Bechgaard salts was criticized by Montambaux 39]. He showed that in that case, a sinusoidal potential induces near the Fermi level a series of Landau bands which are very 
well decribed by the continuum standard model; however, he did not rule out additional effects due to additional harmonics of the periodic potential. The quantization of the Hall effect in case of multiple order parameters has been discussed by Yakovenko in terms of topological invariants of the wave functions [19].

The experimental situation seems to have clarified recently also in this area when thermodynamic measurements with improved accuracy [15] found that the complexity of the phase diagram reduces to a doubling of some transition lines between SDW sub-phases in the $\mathrm{ClO}_{4}$ salt. Most first order transition lines give way to dedoubled second order lines. Simultaneous thermal and transport measurements [16 confirm that all transport transitions (Hall resistance jumps) are associated with thermodynamic transitions. No arborescence of the phase diagram is otherwise observed.

Specifically, in very slowly cooled $\mathrm{ClO}_{4}$ compound, the transitions between FISDW subphases are reproducible and non hysteretic below 6 teslas. Above $6 \mathrm{~T}$ there is a marked hysteresis, and "noise" which differ from field sweep to field sweep.

For $B<6 T$, the transitions between FISDW's appear as transition regions of finite width, in which the Hall resistance changes from one plateau to the next. These rises of the Hall resistance coincide with the magnetocaloric double peaks characterizing these transition regions. The transition regions are about $0.1 \mathrm{~T}$ wide 16$]$. In the $P F_{6}$ compound, transition between plateaux of $\rho_{x y}$ coincide with sharp spikes of $\rho_{x x}$. This behaviour is not that predicted by the QNM, since the latter predicts first order transitions and discontibuous changes of $\rho_{x x}$ with no possibility of pinning the Fermi level except in the middle of a gap. The sharp spikes of $\rho_{x x}$ indicate that the Fermi level crosses a region of extended states, or becomes much closer to one in the transition region. Two reasons can be thought of to produce this: either impurity states, which produce bound states between the Landau bands may pin the Fermi level close to a band edge, or a superposition of two order parameters may result in a magnetization pattern with discommensurations, domain walls and so on. The entrance and the exit of the coexistence region are indeed marked by peaks in $\partial S / \partial B$, where $S$ is the entropy 16.

A phenomenological Landau expansion for a system with two competing order parameter is 16] :36:

$$
f=a_{1}\left(T-T_{c 1}\right) \Phi_{1}^{2}+u_{1} \Phi_{1}^{4}+a_{2}\left(T-T_{c 2}\right) \Phi_{2}^{2}+u_{2} \Phi_{2}^{4}+u_{12} \Phi_{1}^{2} \Phi_{2}^{2}
$$

(a microscopic calculation of coefficients $a_{i}$ and $u_{i}$ is given in [7]). In the absence of the last term this describes, at $T<\left(T_{c 1}, T_{c 2}\right)$ a phase with two noninteracting order parameters. At $T_{c 1}(B)=T_{c 2}(B)$, four transition lines meet. All are second order transition lines and the phase diagram has a tetracritical point, somewhat as observed in $\mathrm{ClO}_{4}$ at $\mathrm{T}=0.67 \mathrm{~K}$ and $H=4.6 T$ [40]. The topology of the phase diagram is unchanged for $u_{12} \geq 0$, as long as $u_{12}^{2}<u_{1} u_{2}$. When $u_{12}^{2}>u_{1} u_{2}$, the stable phases have a single order parameter, and they are separated by a first order transition line. However, the latter may de-double in two second order lines between which two order parameters coexist if, below some temperature $T^{\star}, u_{12}^{2}$ becomes smaller than $u_{1} u_{2}$.

A detailed understanding of the mechanism through which the Fermi level may become pinned in a region of extended states, either because of a superposition of order parameters, or because of impurity states, is still missing. 


\section{Collective Modes of the Ultra Quantum Crystal: the Magneto- Roton of the FISDW Phases}

FISDW phases are similar to conventional SDW phases inasmuch as they possess a spatially periodic magnetization density with wave vector determined by the Fermi surface geometry. The quantization of the Hall effect within each FISDW subphase makes them distinctly different from conventional SDW. Their collective modes also possess specific features which reflect their dual character of electron-hole quantum condensate driven by the electronic orbital motion 20]. Their original properties justify the name "Ultra Quantum Crystal" given to this class of quantum crystal which exist under magnetic field only when $k_{B} T<<\hbar \omega_{c}[20]$.

The derivation of the order parameter collective modes of FISDW phases [20] follows the lines of the derivation by Lee, Rice and Anderson (LRA), of the collective modes in the well known example of 1D CDW 41. The collective modes are obtained by solving, within the Random Phase Approximation, for the poles of the spin-spin correlation function in the ordered phase. Within a weak coupling approximation $\left(\lambda n\left(\epsilon_{F}\right)<<1\right)$, the equation for the collective modes is:

$$
\left(1-\lambda \hat{\chi}_{+-}^{0}\left(\mathbf{Q}_{\mathbf{N}}+\mathbf{q}, \omega\right)\right)\left(1-\lambda \hat{\chi}_{+-}^{0}\left(\mathbf{Q}_{\mathbf{N}}-\mathbf{q}, \omega\right)\right)-\lambda^{2} \Gamma_{+-}^{0}(\mathbf{q}, \omega) \Gamma_{-+}^{0}(\mathbf{q}, \omega)=0
$$

with $\mathbf{q}=\mathbf{Q}-\mathbf{Q}_{\mathbf{N}}=$ collective mode wave vector. In equ.15 $\hat{\chi}_{+-}^{0}$ are the irreducible bubbles renormalized by all possible scatterings on the mean field potentials connected to the various gaps:

$$
\begin{aligned}
\hat{\chi}_{+-}\left(\mathbf{q}, \omega_{p}\right) & =T \Sigma_{n} \int \exp \left[i q_{x}\left(x-x^{\prime}\right)\right] d x \\
<G_{1 \uparrow, 1 \uparrow}\left(p_{\perp}, \omega_{n}, x, x^{\prime}\right) G_{2 \downarrow, 2 \downarrow}\left(p_{\perp}-q_{\perp}, \omega_{n}-\omega_{p}, x^{\prime}, x\right)> &
\end{aligned}
$$

where $\langle\ldots\rangle$ means average on $p_{\perp}, \omega_{n}$ is the Matsubara frequency, and $G_{i \sigma, i \sigma}$ is the Green's function for spin $\sigma$ electrons on the $i$-th side of the Fermi surface. Likewise $\Gamma_{+-}^{0}(\mathbf{q}, \omega)$ is the extraordinary bubble, also renormalized with all possible scatterings. Equ. 15 holds for fluctuations transverse to the order parameter as well as parallel ones, so that the two types of collective modes are degenerate in this approximation.

The simplest approximation resums to all orders the gap $\delta_{N}=\Delta I_{N}$ at the Fermi level and takes all other gaps into account to second order in perturbation. Then

$$
\hat{\chi}_{+-}^{0}\left(\mathbf{Q}_{N}+\mathbf{q}, \omega\right)=\Sigma_{n} I_{N+n}^{2}\left(Q_{\perp}^{N}+q_{\perp}\right) \tilde{\bar{\chi}}^{0}\left(n G-q_{\|}, \omega\right)
$$

and

$$
\Gamma_{+-}^{0}(\mathbf{q}, \omega)=\Sigma_{n} I_{N+n}\left(Q_{\perp}^{N}+q_{\perp}\right) I_{N-n}\left(Q_{\perp}-q_{\perp}\right) \tilde{\tilde{\Gamma^{0}}}\left(n G-q_{\|}, \omega\right)
$$

$\tilde{\bar{\chi}}^{0}$ and $\tilde{\bar{\Gamma}}^{0}$ are for $n=0$ the objects discussed by LRA [41]. For $q x_{0}<<1$ (i.e. $q / G<<1$ ) and $\omega<<\delta_{N}$ equ.15 decouples into phase and amplitude modes:

$$
\left(\omega^{2}-v_{F}^{2} \mathbf{q}^{2}\right)\left(\omega^{2}-v_{F}^{2} \mathbf{q}^{2}-4 \delta_{N}^{2}\right)=0
$$


The (incommensurate) ultraquantum crystal thus exhibits the Goldstone bosons connected to the two broken continuous symmetries: translation symmetry and spin rotational invariance in the spin $(x, y)$-plane. The model also has high frequency amplitude modes. New physics appears for $q_{\|}=m / x_{0}+\delta$, with $\delta x_{0}<<1$ and $m$ integer. In that case, $\hat{\chi}_{+-}^{0}\left(\mathbf{Q}_{N}+\mathbf{q}, \omega\right) \neq \hat{\chi}_{+-}^{0}\left(\mathbf{Q}_{N}-\mathbf{q}, \omega\right)$, so that equ.15 does not factorize anymore.

Then an interaction with the gap at $N \pm m$ allows the collective mode to propagate in a medium almost identical to the case $m=0$ and $q_{\|} x_{0}<<1$. A second interaction allows the outgoing oscillation to retrieve the momentum lost with the first interaction. The mode with $m \neq 0$ would have exactly the same energy as that with $m=0$ and $q_{\|} x_{0}<<1$ if all $I_{N}$ were equal. Such is not the case, so that the phase and amplitude modes of the order parameter are not decoupled anymore for $m \neq 0$ and, instead of a zero energy mode at $q_{\|}=m G$, a local minimum appears. See Figure 5. The collective mode dispersion around the minimum is $\omega_{\text {rot }}\left(q_{\|}\right) \simeq \omega_{\text {rot }}^{0}\left(1+v_{F}^{2} \delta^{2}\right)$, with $\delta=\left(q_{\|}-G\right)$. The dispersion relation in the transverse direction is determined by the $q_{\perp}$ dependance of the $I_{N}$ coefficients in equ.5 and is therefore much smoother than along the $q_{\|}$direction: the magneto-roton dispersion relation is very anisotropic.

The location of the magneto-roton minimum within the single particle gap was shown graphically to vary with field and temperature within a sub-phase 20]. Close to a transition line between two sub-phases (assuming single Fourier component order parameters) $\omega_{\text {rot }} \leq$ $\sqrt{2} \delta_{N}(T)$. As T decreases from $T_{c}^{N}$, the relative distance $\left(2 \delta_{N}-\omega_{\text {rot }}\right) / 2 \delta_{N}$ increases. Other roton-like minima may exist for wave vector with $q_{\|}=(2,3$, etc $) G$, but no rigorous proof was given; their energy in any case is larger than for $q_{\|}=2 \pi / x_{0}=G$.

To summarize, the magneto-roton of the FISDW, derived within the QNM, is a specific signature of the ultra quantum crystal, as discussed in ref. [20]. There is a close analogy between the FISDW, the Fractional Quantum Hall Effect 43 and superfluidity, the common factor being the absence of low-lying single-particle excitations, and the existence of a collective mode energy minimum at a finite wave vector. The latter, in superfluid HeII 44], in the FQHE[3], and in FISDW, is determined by the lattice parameters of a neighbouring competing phase: the quantum crystal in the case of HeII, the Wigner crystal in the FQHE, the phases with $N^{\prime}=N \pm m$ in the FISDW case. All three systems exhibit elementary particle excitations consisting of phonons (phasons or magnons in FISDW) and rotons. In addition, HeII and FQHE have quantized vortices. FISDW also have topological excitations characteristic of quantum antiferromagnets, skyrmions, or half skyrmions, as discussed later. The latter are vortices when the easy plane is orthogonal to the magnetic field, as assumed in this paper [19]. In the FQHE, phonons have a "mass", in contrast with the situation in HeII, or with the phasons in FISDW. In FISDW, the magneto-roton is anisotropic, in contrast with HeII and the FQHE.

\subsection{Experimental evidence for the magneto-roton}

The magneto-roton of FQHE states, as specific manifestation of the quantum Hall condensate has stimulated a number of successful experimental investigations 45]. So far the magneto-roton in FISDW has attracted little interest, and no experimental work has been explicitly conducted to prove or disprove its existence. This is understandable in view of the practical difficulty (low temperatures, high fields) and in view of the doubts raised until recently about the validity of the mean field picture set up by the QNM.

The time may have come for more active investigations of this aspect of the FISDW 
physics, both on the theory side and on the experimental one. Indeed, I believe that the signature of the magneto-roton was indeed observed 446] some years ago, although it was not identified as such. In contrast with the FQHE case, thermodynamic measurements are good candidates for the experimental investigation of low energy excitations, since samples are macroscopic three dimensional ones, in contrast with the $2 \mathrm{D}$ inversion layers of the FQHE. (In this respect the low temperature thermodynamics of the Quantum Hall Effect system in FISDW may prove much more interesting and easier to reach experimentally with thermodynamic measurements than in the 2D FQHE system). Neutron inelastic scattering, which has been so decisive in the observation of rotons in HeII [44] are of little use, at first sight, in FISDW, because of the smallness of the order parameter.

Consider first, for simplicity, the consequences of the magneto-roton on the low temperature specific heat of FISDW in a one dimensional picture. In a temperature interval $T_{c}^{N}>T>\omega_{\text {rot }}$ the free energy is dominated by single particle excitations accross the gap, and varies roughly as in a conventional BCS s-wave superconductor; thus $C_{p} \propto \exp -\delta_{N} / T$. In that temperature interval, the contribution of magneto-rotons to the free energy is negligible: $\delta F_{\text {rot }} \simeq \Sigma_{q} \omega_{\text {rot }}(q) n_{B}\left(\omega_{\text {rot }}(q)\right)$, where $n_{B}$ is the Bose occupation factor. Apart from small corrections, in that temperature interval, $\delta F \simeq T$. However, when $T<\omega_{\text {rot }}$, the specific heat is eventually dominated by the magneto-roton gap, not by the single particle gap any more. Indeed, $\delta F_{\text {rot }} \simeq \sqrt{k_{B} T \omega_{\text {rot }}}\left(\omega_{\text {rot }} / \epsilon_{F}\right) \exp -\frac{\omega_{\text {rot }}}{k_{B} T}$, so that the specific heat is $C_{p} \simeq \frac{k_{B} T}{\epsilon_{F}}\left(\frac{\omega_{r o t}}{k_{B} T}\right)^{7 / 2} \exp -\frac{\omega_{r o t}}{k_{B} T}$. At lower temperature yet, $C_{p} \simeq k_{B} T / \epsilon_{F}$. Obviously, the same qualitative behaviour prevails in three dimensions, where the prefactors of the exponentials only are different.

Specific heat measurements in $(\mathrm{TMTST})_{2} \mathrm{ClO}_{4}$ at 10 Tesla from $\mathrm{T}=T_{c}$ down to $T=.4 T_{c}$ seem to exhibit precisely this behaviour [42]: below $T \simeq 0.8 T_{c}$ an exponential behaviour is observed for the specific heat as a function of $T$, and, below $T \simeq 0.6 T_{c}$, a different slope of $L n C_{p}$ vs $T_{c} / T$ sets in, corresponding to a smaller gap; the ratio of the two gaps is about 1.5 , which is quite a reasonable value for a ratio $\Delta / \omega_{\text {rot }}$. See Figure 6. Unfortunately, this behaviour has not been studied systematically, and more data, at different fields, and lower temperatures are clearly needed. As discussed in detail in ref 20], the QNM offers specific predictions on the evolution of $\omega_{\text {rot }}$ with field and temperature, which are connected with the virtual transition temperatures $T_{N \pm m}$ which form a network of lines in the $(\mathrm{T}, \mathrm{H})$ phase diagram, within a given $\mathrm{N}$ subphase 32]. The low temperature power law behaviour due to the conventional spin waves and phason modes has not been observed, most likely for want of low enough temperatures. Notice that pinning effects will result in a phason mode gap at long wavelength, and spin anisotropy in a spin wave gap at $q=0.47$

Among other experimental tests and consequences of the existence of magneto-rotons, one may think of Raman experiments, phonon scattering experiments, etc.. Simple transport properties such as determinations of $\rho_{x x}$ as a function of field and temperature may also yield interesting results. Suppose that the electronic relaxation time is dominated by inelastic scattering off the magneto-roton modes, i.e. $\tau_{e l}>>\tau_{\text {inel }}$, (where $\tau_{e l}$, resp. $\tau_{\text {inel }}$, is the elastic, resp. inelastic, electronic transport lifetime). I assume that the temperature range is such that $\tau_{\text {inel }}$ is essentially limited by electron-magnetoroton collisions. Then, if $k_{B} T<\hbar \omega_{\text {rot }}\left(\delta=q_{\perp}=q_{z}=0\right), \tau_{\text {inel }} \propto \exp \left(\omega_{\text {rot }} / k_{B} T\right)$. This behaviour is likely to be obtained in a sizeable temperature interval. At lower temperatures,()below a temperature $\theta^{*}$ collisions with the linear-in-q branch of the collective 
modes may overcome the magneto-roton contribution, and elastic scattering processes will ultimately dominate at the lowest temperatures.However, above $\theta^{*}$ one should observe $\rho_{x x} \propto \exp -\left(\left(\Delta(T, H)-\hbar \omega_{\text {rot }}(T, H)\right) / k_{B} T\right)$. Combined to specific heat measurements, this might allow a determination of $\omega_{\text {rot }}(T, H)$ at least for temperatures below $\hbar \omega_{c} / k_{B}$ and above $\theta^{*}$.

\section{The Skyrmions and Half Skyrmions}

The material in this section relies mostly on the paper by Yakovenko (ref. [19]) Call $\mathbf{n}$ the unit vector in the direction of the SDW order parameter. The effective action of the $\mathbf{n}$ field, which may vary slowly in space and time $(x, y, t$,$) can be found, after integrating$ the fermions out of the action, as a series in powers of gradients of $\mathbf{n}$. Apart from the standard term $\propto(\nabla \mathbf{n})^{2}$, it may contain the topologically non trivial Hopf term [48]:

$$
\begin{aligned}
S_{H} & =\frac{C \epsilon_{\mu \nu \lambda}}{32 \pi} \int d x d y d t A_{\mu} F_{\nu \lambda} \\
F_{\mu \nu} & =\mathbf{n}\left(\partial_{\mu} \mathbf{n} x \partial_{\nu} \mathbf{n}\right), \\
\partial_{\mu} A_{\nu}-\partial_{\nu} A_{\mu} & =F_{\mu \nu},
\end{aligned}
$$

Here $\mu=t, x, y$ and $\epsilon_{\mu \nu \lambda}$ is the completely antisymmetric Levi-Cività tensor of rank 3 . The coefficient $C$ in equ. 20 determines the spin and statistics of the particlelike topological solitons of the $\mathbf{n}$ field, called skyrmions [49]. In zero external magnetic field, the skyrmion has $\mathbf{n}$ up at infinity, down in the center of the skyrmion, and there is a concentric domain wall in between, where $\mathbf{n}$ rotates between up and down direction.

Volovik and Yakovenko [50] have shown that the value of the coefficient $C$ in equ. 20 has by the same expression as in the Hall conductivity:

$$
\sigma_{x y}=C e^{2} / h
$$

As a result, in a FISDW subphase with quantum number $N, C=2 N$. Following ref. 449], this means the skyrmions are bosons with integer spin $N$. In fact, if the magnetic field is taken into account, two situations may arise, depending on whether the order parameter is transverse to the external field ( I have considered only this situation here), or along the field $[19]$. In the former case $(\mathbf{n} \perp \mathbf{H})$, the topological excitations are half skyrmions, with spin $N / 2$ and corresponding statistics; they are vortices with $\mathbf{n} \| \mathbf{H}$ in the vortex core.

\section{Conclusion}

I have left aside a number of open problems which are not (or so it seems) directly connected with the topic of this review: the "magic angle" problem set up by Lebed [51], which may have fascinating implications for the discussion of the non Fermi liquid ground state of quasi 1D conductors [21] [52]. This problem arises when the magnetic field deviates from the direction orthogonal to the most conducting plane, a situation I have not tackled here. Other problems, which are still under discussion, the fast oscillation problem, or the normal state magneto-resistance, do not seem to have obvious implications for the understanding of the Quantum Hall Effect. 
This review has focused on the reasons for renewed confidence in the usefulness of the Quantized Nesting Model characterized by a FISDW order parameter with a single Fourier component of the magnetization. The main recent new facts in this respect are:

- The experimental observation [13] of a Ribault anomaly all the way from the normal phase down to low temperature, with a well identified critical line separating the normal phase from the $N=-2$ FISDW. This observation establishes that the Ribault anomaly is connected with the instability mechanism of the normal phase, not with a superposition of order parameters.

- The simple and elegant theoretical interpretation of this observation with the introduction in the usual QNM of additional higher order perturbative terms in the electronic dispersion law of the normal phase [17]. This allows to describe the normal phase instability leading to a succession of FISDW subphases exhibiting the Ribault anomaly phenomenon.

- The re-interpretation of old specific heat data 42 which may well be the first experimental observation of a magneto-roton in FISDW, or, for that matter in the specific heat of a Quantum Hall Effect system [46]. This interpretation calls for a new experimental effort in determining the low temperature specific heat of FISDW phases. Other experimental techniques, such as Raman spectroscopy[53], non-equilibrium phonon absorption [45] might also prove very useful for direct observation of the magneto-roton.

In view of this standpoint, I have not devoted a lot of space to the discussion of the physics of multiple order parameters; this does not mean that this problem is altogether devoid of interest. In particular, the recent findings about the de-doubling of the sub-phase to subphase transition line very likely indicates that mixtures of order parameters are at work, although this looks more like a mechanism for the destruction of the Quantum Hall Effect. In fact, one of the open questions is the mechanism of dissipation leading to spikes in $\rho_{x x}$, as observed by Balicas et al. [13], in a way similar to the usual situation in the QHE, and in contrast to the behaviour suggested by the QNM when no mixing of Fourier components is considered. A possibility is that this mixture of order parameters helps pinning the Fermi level in, or close to, a region of extended states. In fact, the low temperature behaviour of $\rho_{x x}$ is poorly known. It is not clear that it vanishes exponentially with temperature 54 The role played by phase defects of the FISDW under the action of pinning centers, as well as the consequences on the dissipation $\left(\rho_{x x}\right)$ of low lying collective modes within the single particle gap may lead to significant deviations from the physics of the FQHE at low temperature.

Should the success of the QNM lead one to the notion that it proves the Fermi liquid nature of the ground sate in the anisotropic metallic organics in the absence of magnetic field? Although I have argued that it is likely that below $T \simeq t_{c} / k_{B}$ the ground state is indeed an anisotropic Fermi liquid, I do not consider this point to be settled by the success of the weak coupling theory. All I can say is that it would be very unlikely o obtain the description of the cascade of Quantum Hall States in the absence of a zero field Fermi surface. However, the nature of excitations around this Fermi surface, conventional quasi-particles with life time $\propto T^{-2}$ or spin charge separated many body states such as spinons and holons 55] leading to a Luttinger liquid cannot be settled. I cannot exclude 
the possibility that a strong coupling theory, based on spin charge separation, eventually sets up an equally successful theoretical picture. Indeed, a mean field strong coupling theory of the normal state in two dimensions describes de Haas van Alphen oscillations with the same frequency, and in general, the same qualitative behaviour as non interacting Fermi gas 56 .

This last remark indicates that lively controversies about the Quantum Hall Effect of the Ultra Quantum Crystal are still to be expected ahead of us!

Acknowledgements. I would like to thank Luis Balicas, Serguei Brazovski, Claude Pasquier, Heinz Schulz, Drazen Zanchi, Patrick Lee, for useful discussions. I am grateful to Gilles Montambaux for a careful reading of the manuscript. 


\section{References}

[1] M. Ribault,D. Jérôme, J. Tuchendler, C. Weyl, and K Bechgaard, J. Physique Lett.44, L 953, (1983). P. M. Chaikin e al., Phys. Rev. Lett., 51, 2333, (1983).

[2] J. F. Kwak, J. Schirber, R. L. Greene and E. Engler, Phys. Rev. Lett., 46, 1296, (1981); J. F. Kwak, J. Physique Colloq. 44, C3-839, (1983)

[3] The Quantum Hall Effect, R. E. Prange, S. M. Girvin ed., Springer Verlag, (1987). Chap IX, by S. Girvin, focuses on collective excitations in the QHE.

[4] G. Montambaux, Physica Scripta, T 35, 188, (1991).

[5] L. P. Gor'kov and A. G. Lebed, J. Physique. Lett. 45, L433, (1984).

[6] M. Héritier, G. Montambaux and P. Lederer, Jour Physique Lett. 45, L943, (1984). See also K. Yamaji, J. Phys. Soc. Jap. 54, 1034, (1985) and K. Maki, Phys. Rev.B 33, 4826, (1986).

[7] D. Poilblanc, M. Héritier, G. Montambaux, and P. Lederer, J. Phys. C Sol.St. Phys. 19, L321, (1986). See also G. Montambaux and D. Poilblanc, Phys. Rev. B 37, 1913, (1988).

[8] A. G. Lebed, Sov. Phys. JETP, 62, 595, (1985).

[9] B. I. Halperin, Jpn. J. Appl. Phys. Suppl.3, 26,1913, (1987 ).

[10] P. Garoche, R. Brusetti, D. Jérôme and K. Bechgaard, J. Physique Lett. 43, L147, (1982). T. Takahashi, D. Jérôme, and K. Bechgaard, J. Physique Lett. 43, L565, (1982).P. Garoche, R. Brusetti and K. Bechgaard, Phys. Rev. Lett. 49, 1346, (1982). M. J. Naughton, Phys. Rev. Lett. 55, 969, (1985). F. Pesty,P. Garoche and K. Bechgaard, Phys. Rev. Lett. 55, 2495, (1985).

[11] J. R. Cooper, W. Kang, P. Auban, G. Montambaux and K. Bechgaard, Phys. Rev. Lett. 63,1984, (1989); S. T. Hannahs, J. S. Brooks, W. Kang, L. Y. Chiang, and P. M. Chaikin, Phys. Rev. Lett. 63, 1988, (1989). The following reference s deal with the QHE in $\mathrm{TMTSF}_{2} \mathrm{ClO}_{4}$ : R. V. Chamberlin, M. J. Naughton, X. Yan, L. Y. Chiang, S.-Y. Hsu, and P. M. Chaikin, Phys. Rev. Lett. 60, 1189, (1988). M. J. Naughton, R. V. Chamberlin, X. Yan, S.-Y. Hsu, L. Y. Chiang, M. Ya. Azbel, and P. M. Chaikin, Phys. Rev. Lett. 61, 621, [1988].

[12] M. Ribault,Mol. Cryst.Liq. Cryst. 119, 91, (1985).

[13] L. Balicas, G. Kriza and F. I. B. Williams, Phys. Rev. Lett. 75, 2000, (1995). The detailed observation of the Hall effect sign inversion by Balicas, Kriza and Williams was made possible through a remarkable improvement of the stability of the observed phases, by shaking down the phase defects of the FISDW order parameter responsible for the observed global hysteresis: application of an electric field pulse larger than the depinning field allows to anneal the modulated phase and reduces the hysteresis by an order of magnitude. 
[14] F. Pesty and P. Garoche, Fizika (Zagreb), 21, 40, (1989); F. Pesty, P. Garoche, and M. Héritier, in The Physics and Chemistry of Organic Conductors, edited by G. Saito and S. Kagoshima, Springer Proceedings in Physics, Vol. 51, (Springer, Berlin, 1990); G. Faini, F. Pesty and P. Garoche, J. Phys. (Paris) Colloq. 49, C8-807, (1988)

[15] U. M. Scheven, W. Kang and P. M. Chaikin, Jour. Physique IV C2, 3, 287, (1993).

[16] U. M. Scheven, E. I. Chashechkina, E. Lee, and P. M. Chaikin, Phys. Rev. B52, 3484, (1995)

[17] D. Zanchi and G. Montambaux, to be published

[18] K. Machida, Y. Hori and M. Nakano, Phys. Rev. Lett. 70, 61, (1993). K. Machida, Y. Hasegawa, M. Kohmoto, V. M. Yakovenko, Y. Hori, and K. Kishigi, Phys. Rev. B 50, 921, (1994)

[19] V. M. Yakovenko, Phys. Rev. B 43, 11 353, (1991).

[20] P. Lederer and D. Poilblanc, C. R. Acad. Sc. Paris, 304, II-251, (1987). D. Poilblanc and P. Lederer Phys. Rev. B 37, 9650, (1988); B 37, 9672, (1988)

[21] S. P. Strong, D. G. Clarke and P. W. Anderson, Phys. Rev. Lett. 73, 1007, (1994)

[22] K. Yamaji, J. Phys. Soc. Japan 51, 2787, (1982).

[23] K. Yamaji, J. Phys. Soc. Japan 55, 860, (1986); 56, 1841, (1987).

[24] A. Virosztek, L. Chen and K. Maki Phys. Rev. B 34, 3371 (1986).

[25] D. Poilblanc, G. Montambaux, M. Héritier, and P. Lederer, Phys. Rev. Lett.58, 270, (1987).

[26] P. St $\breve{r}$ eda, J. Phys. C15, L1299, (1982)

[27] D. J. Thouless, M. Kohmoto, M. P. Nightingale, and M. den Nijs, Phys. Rev. Lett.49, 405, (1982)

[28] W. Kang, S. T. Hannahs, and P. M. Chaikin, Phys. Rev.Lett. 70 , 3091, (1993).

[29] L. Brossard et al., Physica (Amsterdam), 143B, 406, (1986)

[30] W. Kang, J. R. Cooper and D. Jérôme, Phys. Rev. B 43 , 11467, (1991).

[31] G. Montambaux, M. Héritier, and P. Lederer, Phys. Rev. Lett. 55, 2078, (1985)

[32] P. Lederer, D. Poilblanc and G. Montambaux Europhys. Lett. 2, 151, (1988)

[33] G. Montambaux, Thèse d'Etat, Orsay, (1985), G. Montambaux, NATO ASI on Low dimensional conductors and superconductors, Vol. 155, p.233, D. Jérôme and L. Caron eds. (Plenum, New York 1986)

[34] P. Lederer and G. Montambaux, Synthetic Metals, 27, A147, (1988).

[35] U. M. Scheven, E. I. Chashechkina, E. Lee, and P. M. Chaikin, Phys. Rev. 52, 3484, (1995) 
[36] A. Lebed, JETP Lett. 51, 663, (1990).

[37] F. Pesty and P. Garoche, Fizika (Zagreb), 21, 40, (1989); F. Pesty, P. Garoche, and M. Héritier, in The Physics and Chemistry of Organic Conductors, edited by G. Saito and S. Kagoshima, Springer Proceedings in Physics, Vol. 51, (Springer, Berlin, 1990); G. Faini, F. Pesty and P. Garoche, J. Phys. (Paris) Colloq. 49, C8-807 (1988)

[38] D. R. Hofstadter, Phys. rev.. 14, 2239, (1976)

[39] G. Montambaux, Synthetic Metals, 41-43, 3807, (1991)

[40] F. Tsobnang, F. Pesty, P. Garoche, and M. Héritier, Synth. Metals, 41-43, (1991).

[41] P. A. Lee, T. M. Rice and P. W. Anderson, Sol. St. Com. 14, 703, (1986).

[42] F. Pesty, P. Garoche, and M. Héritier, Mat. Res. Soc. Symp. Proc. 173, 205, (1990);

F. Pesty, Thèse d'habilitation, Université Paris-Sud, Orsay,P-13, p. 205 (1994)

[43] S. M. Girvin, A. H. MacDonald and P. M. Platzman, Phys. Rev. B 33, 2481, (1986).

[44] See for example, Superfluidity and Superconductivity, by D. R.Tilley and J.Tilley, Adam Hilger Ltd, (1990)

[45] See for example, among recent work C. J. Mellor, J. E. Digby, R. H. Eyles, A. J. Kent, K. A. Benedict, L. J. Challis, M. Henini, C. T. Foxon, J. J. Harris, Physica B 211, 400, (1995).

[46] P. Lederer, (unpublished)(1996)

[47] Remember I am discussing here an order parameter with no component along the external field. Spin waves with spin $S=1$ have one branch with $S_{z}=0$ which have no Zeeman gap. I am indebted to Heinz Schultz for a remark on that point.

[48] I. Dzyaloshinskii, A. Polyakov, and P. Wiegmann, Phys. Lett. A 127, 112, (1988)

[49] F. Wilczek and A. Zee, Phys. Rev. Lett.51, 2250, (1983)

[50] G. E. Volovik and V. M. Yakovenko, J. Phys. Condens. Matter 1, 5263, (1989); V. M. Yakovenko, Phys. Rev. Lett. 65, 251, (1990)

[51] A. G. Lebed, JETP Lett.43, 174, (1986)

[52] G. M. Danner and P.M. Chaikin, Phys. Rev. Lett, 75, 4690, (1995)

[53] I am indebted to Patrick Lee for this suggestion.

[54] . D. Jérôme, private communication.

[55] P. W. Anderson, Science, 235, 1196, (1987)

[56] J. P. Rodriguez and Pascal Lederer, Int. Jour. Mod. Phys. B 6, 49, (1992) 
Figure Captions

Fig1. Experimental QHE of $(T M T S F)_{2} P F_{6}$ under a pressure of 9kbar. The measured resistance is multiplied by the number of conducting layers to obtain $\rho_{x y}^{2 D}$, and then renormalized to the value of the resistance quantum. The insert exhibits the experimental set-up for the 8 electrical contacts.(courtesy of Luis Balicas, thèse, 1995)

Fig. 2 The Ribault anomaly observed by Balicas et al. under a pressure of $8.5 \mathrm{kbar}$ (after ref. [13]) The sign change is observed all the way down from the normal phase, along a critical line with about $0.2 \mathrm{~T}$ length along the magnetic field axis.

Fig.3 Staggered Spin Susceptibility in the normal phase in the presence of a magnetic field, at fixed $q_{z}=\pi / c$ (after ref. [31]. A series a peaks appear under field parallel to the $\mathrm{c}$ axis. The peaks have a quantized component along the a axis. As the field varies, the peak intensities varies, and the absolute maximum shifts dicontinuously from one peak to the other.

Fig. 4 Staggered Spin susceptibility in the normal phase, in the presence of a field, when additional perturbative terms are taken into account $\left(t_{3} a n d T_{4} \neq 0\right.$ (after ref. [17]). a)Here $t_{b}^{\prime}=10 K, t_{3}=t_{4}=0$. The best nesting vector is $Q^{*} . Q^{0}$ is a degenerate secondary maximum. b) Same parameters. c) Afinite $t_{3}=10 \mathrm{~K}$ alters the best nesting and $Q^{0}$ is now the degenerate best nesting vector. d) A finite $t_{4}=0.2 \mathrm{~K}$ lifts this degeneracy, leading to a negative quantum number.

Fig. 5 The magneto-roton dispersion relation of the Ultra Quantum Crystal (after ref. [20]) The minimum shown is in the $q \|$ direction. The effective mass in the $q_{\perp}, q_{z}$ directions is much smaller.

Fig.6 The specific heat at low $\mathrm{T}$ and $\mathrm{H}=10 \mathrm{~T}$ (after ref. 42]). The exponential decrease of $C_{e l}$ below the metal-FISDW transition results from the opening of an energy gap at $\epsilon_{F}$. Two gaps are extracted from the two slopes indicated on the figure. The first one (about 1.5 times the BCS value) corresponds to the single particle gap. The second one, about .6 times the value of the single particle gap, is, in my view, the magneto-roton gap. 
Running title

The Quantum Hall Effect of FISDW Phases 